\title{
Design data for quick development of folded $H$ plane tee at high average power level
}

\author{
HARISH V DIXIT ${ }^{1, *}$, AVIRAJ R JADHAV ${ }^{2}$, YOGESH M JAIN ${ }^{3}$, ALICE N CHEERAN ${ }^{1}$, \\ VIKAS N GUPTA ${ }^{2}$ and P K SHARMA ${ }^{3}$ \\ ${ }^{1}$ Veermata Jijabai Technological Institute, Mumbai 400019, India \\ ${ }^{2}$ Vidyavardhini's College of Engineering and Technology, Vasai 401202, India \\ ${ }^{3}$ Institute for Plasma Research, HBNI, Gandhinagar 382428, India \\ e-mail: hvdixit48@yahoo.com
}

MS received 17 July 2016; revised 17 February 2017; accepted 31 August 2017; published online 14 March 2018

\begin{abstract}
At high average power level, waveguide-based structures are indispensable in microwave systems due to their higher power handling capacity. These structures are often used to perform power division and combination operation. Folded $\mathrm{H}$ plane tee is one of the many components that can be used for the power division application. Its advantage is that the output arms and input arms are in a single plane along a single axis, thus taking less space in the overall system. However, their proper thermal management is indispensable for its use, as high surface temperature of the waveguide reduces its power handling capacity at high power level. This paper presents quick, easy design steps and methodology for obtaining the structure of a folded $\mathrm{H}$ plane tee at any given frequency of operation. Further, a scheme for the thermal management for high input power level (500 kW continuous wave) is presented. The proposed methodology is tested at various frequencies in COMSOL Multiphysics, CST Microwave Studio and HFSS with excellent results. A prototype structure was fabricated for use at $3.7 \mathrm{GHz}$, which exhibited good agreement with the simulation results.
\end{abstract}

Keywords. Design methodology; microwave devices; stress; thermal management; waveguide junction.

\section{Introduction}

Waveguide power dividers find many applications in microwave systems. These structures are indispensable in high-power applications like accelerators and heating systems for tokamaks in plasma-related experiments and applications. One such application is feeding the poloidal sections of a Passive Active Multijunction Antenna (PAM) $[1,2]$. This type of antenna is a structure based on phased waveguide array used to drive power at a low hybrid frequency in the tokamak so as to sustain plasma discharges for long periods [3, 4]. A PAM antenna is being designed for the ADITYA-U Tokamak at the Institute for Plasma Research, Gandhinagar. This PAM antenna will have two modules, each having two active and two passive guidelets in the toroidal direction. There will be two poloidal sections of such toroidal rows. As the proper phasing is essential to maintain the directivity of the antenna, a single input from the klystron (TH-2103D) capable of supplying $500 \mathrm{~kW}$ continuous wave $(\mathrm{CW})$ power is given, which is then further divided and fed into the individual poloidal sections of the PAM antenna. More literature for various design

*For correspondence considerations of the PAM antenna can be found in [1]. The conventional $\mathrm{E}$ and $\mathrm{H}$ plane power divider has all its three ports in different directions, thus consuming a lot of space. A folded tee or power divider, on the other hand, has a compact structure and thus can be assembled and integrated easily with other components. It is thus decided to incorporate a folded $\mathrm{H}$ plane tee (FHT) to split the input power from the klystron into two parts so as to feed the two poloidal sections of the PAM antenna.

Even though a few exact analysis techniques [5-7] exist for the evaluation of these type of structures, designing a power divider from these techniques is often cumbersome and requires excessive EM simulation, for which one has to have access to computational power. In [8], detailed design data for various waveguide structures are provided. However, generating a design using these design data would require a recourse to lot of computation. As such, the objective of this work is to present a novel design methodology for the quick development of $\mathrm{H}$ plane folded power divider. As this structure is to be used at a high $\mathrm{CW}$ power level, its thermal management is crucial so as to avoid breakdown due to heating on account of high average power. Thus, another objective of this paper is to present a scheme for the thermal management of the designed structure. As per the best of knowledge of the authors, this 
is the first work that provides closed-form expressions for various parameters to design an FHT. Further data on various aspects for the thermal management of the structure are presented. The design methodology developed and the resultant data can be used to obtain all the required dimensions of the power divider by hand calculations.

The remainder of the paper is organised as follows. Section 2 discusses the proposed geometry and the design steps. Section 3 gives the methodology in detail for a particular case of designing in $\mathrm{S}$ band. Section 4 discusses the $\mathrm{RF}$ results along with the generalization for any frequency of operation. Section 5 explains the thermal management of the structure while section 6 discusses the prototype fabrication and experimental results. Section 7 concludes the paper.

\section{Geometry and design methodology}

Figure 1a shows the 3D structure of the power divider, while figure $1 \mathrm{~b}$ shows the top view of the geometry and the labels assigned to various parts of the folded power divider proposed in this paper. The folded power divider comprises of four units. The first unit is the input section, which can be any standard waveguide depending on the frequency of operation to ensure dominant mode of propagation. The second is a tapered unit, such that its broader waveguide dimension at one side is the same as that of the input section and on the other side is $a_{-} j n$. The length of the tapered section is selected such that the reflections arising at the interface $1-1^{\prime}$ and $2-2^{\prime}$ in figure $1 \mathrm{~b}$ cancel each other. The propagation of the wave in the transition region is still in the dominant mode, as no discontinuity or transitions have been encountered by it. One side of the interface $3-3^{\prime}$ is the transition unit, while the other is the third unit called output unit with width $a_{-} j n$. The $a_{-} j n$ is selected in a way that it allows, along with the dominant mode, the formation of $\mathrm{TE}_{20}$ mode while suppressing all other higher modes.

The fourth section is the output unit consisting of two collinear arms, each having a width of $a_{-}$coll. The electric field is forced to be zero so as to satisfy the boundary condition at the intersection of the interface $3-3^{\prime}$ and $4-4^{\prime}$ due to the presence of the conductor. Thus the field at the interface $3-3^{\prime}$ consists of two half cycles, thereby resembling the $\mathrm{TE}_{20}$ mode. Each of these half cycles further propagates in the two arms of the output unit. An impedance matching at $3-3^{\prime}$ is essential so as to not have any reflection from this interface. As such, the impedances of the transition unit and the output unit have to be equal. It has to be noted that the wave resembles the $\mathrm{TE}_{20}$ mode at transition unit side of the interface, while it is in the dominant mode in each of the arms of the output unit. Both the arms of the output unit appear to be in parallel when seen from the interface. Thus the parallel combination of $\mathrm{TE}_{10}$ impedance of the output arms must be equal to the $\mathrm{TE}_{20}$ mode impedance of transition unit. This then determines $a \_c o l l$.

One dependent dimension is the thickness of the partition th_par, which is fixed once $a \_j n$ and $a \_c o l l$ are determined. The length of the input, transition and output units is $L$ while the length $d$ of the tapered unit is to be determined by an appropriate matching algorithm. The narrow dimension of the power divider is uniform throughout and is the same as that of the standard height of the input waveguide unit.

\section{Illustration}

An illustration of the design of the power divider using the methodology described in section 2 is now provided. A case of designing a power divider at $3.7 \mathrm{GHz}$ is considered.

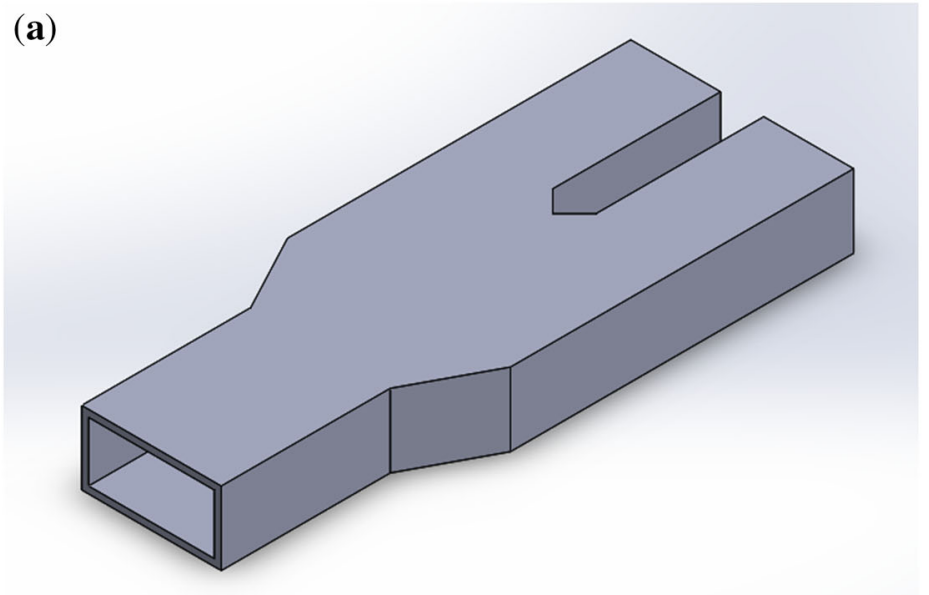

(b)

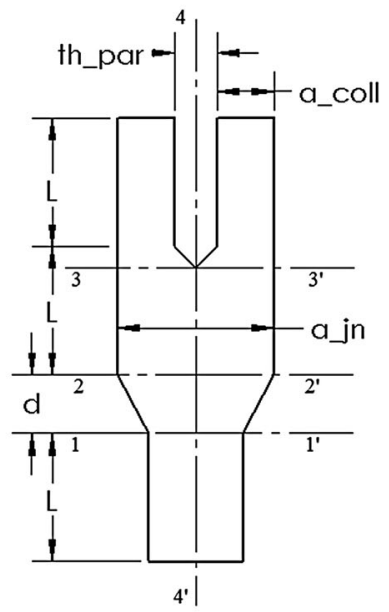

Figure 1. (a) 3D structure of the FHT and (b) top view of the FHT. 


\subsection{Selection of standard waveguide}

The commercially used standard waveguide pertaining to the frequency of $3.7 \mathrm{GHz}$ is WR-284 having the broader dimension ' $a$ ' equal to $72.136 \mathrm{~mm}$ and the narrower dimension ' $b$ ' equal to $34.036 \mathrm{~mm}$.

\subsection{Selection of $a \_j n$}

As described in section 2, $a_{-} j n$ should be selected such that the propagation of $\mathrm{TE}_{20}$ mode is facilitated while that of the $\mathrm{TE}_{30}$ mode is suppressed. For this, the broader dimension of the waveguide must be approximately between 82 and $121 \mathrm{~mm}$. A plot of $S_{21}$ and $S_{31}$ of the power divider considering various values of $a_{-} j n$ in this interval is shown, respectively, in figure $2 \mathrm{a}, \mathrm{b}$. If the $a_{-} j n$ is selected closer to the upper limit, the power is divided equally with $S_{21}$ and $S_{31}$ closer to $-3 \mathrm{~dB}$. This is also true in the case of any frequency at any arbitrary standard waveguide. As such, in this case, $a_{-} j n$ is selected to be $121 \mathrm{~mm}$ (which is $1.4923 \lambda$ at $3.7 \mathrm{GHz}$ ) where $\lambda=c / f, c$ is the speed of light in vacuum and $f$ is the frequency of operation.

\subsection{Selection of $L$ and taper length}

The length of the input, transition and output unit is $L$. A study of effect of variation of $L$ on $S_{21}, S_{31}$ and $S_{11}$ of the power divider for a fixed $a_{-} j n$ (here $a_{-} j n=121 \mathrm{~mm}$ ) was carried out. The results are presented in figure 3 . It is to be noted that the variations in $S_{21}$ and $S_{31}$ are negligible and both remain to be near $-3 \mathrm{~dB}$ value as the length $L$ changes. But there exists a certain value of $L$ for which a minimum value of $S_{11}$ is obtained. In the present case, this happened when $L$ is $100 \mathrm{~mm}$ (which is $1.2333 \lambda$ at $3.7 \mathrm{GHz}$ ). Hence, a small parametric sweep of $L$ can be carried out after fixing the value for $a_{-} j n$ to obtain the minimum $S_{11}$ for any designing frequency. Next, the length of the tapered section is selected to minimize the reflection due to transition. This length is obtained by solving Eq. [9]

$$
d=\frac{n\left(a_{1}-a_{0}\right)}{\left(\frac{2 a_{1}}{\lambda_{g 1}}-\frac{2 a_{0}}{\lambda_{g 0}}\right)-\cos ^{-1}\left\{\frac{\lambda^{2}}{4 a_{0} a_{1}}\left[1+\frac{2 a_{1}}{\lambda_{g 1}} \frac{2 a_{0}}{\lambda_{g 0}}\right]\right\}}
$$

where $a_{1}=a_{\_} j n$ is the broader dimension of the transition/ junction section, $a_{0}=a$ is the broader dimension of the input, $\lambda_{g 1}$ is the guided wavelength in transition section, $\lambda_{g 0}$ is the guided wavelength in input section and $n$ is any natural number $(1,2,3, \ldots)$ (here, $n=1)$.

\subsection{Determining the dimension of output arms}

Modal impedance of an air-filled waveguide with propagating $\mathrm{TE}_{m 0}$ mode is given by [10] the relation
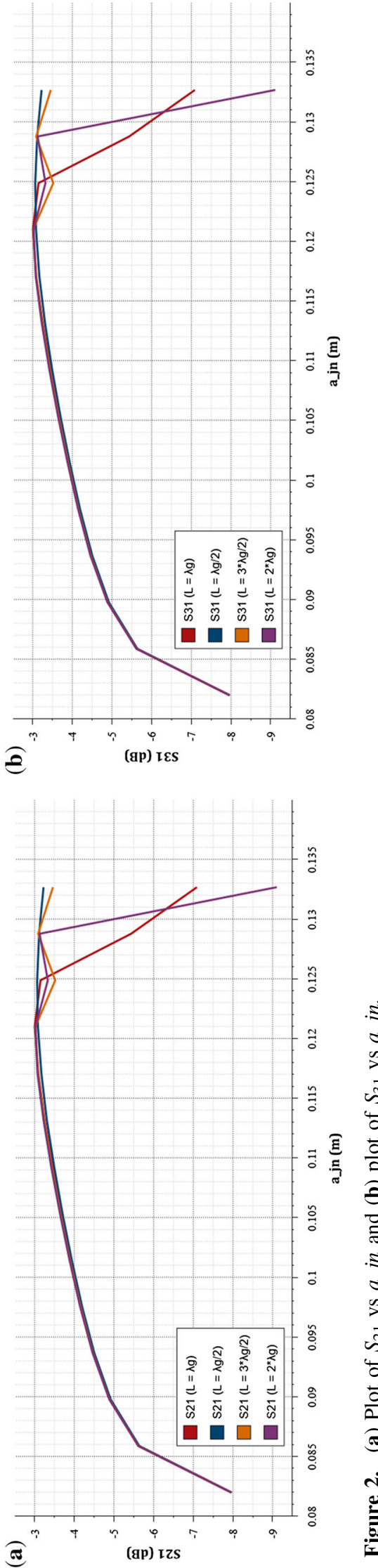


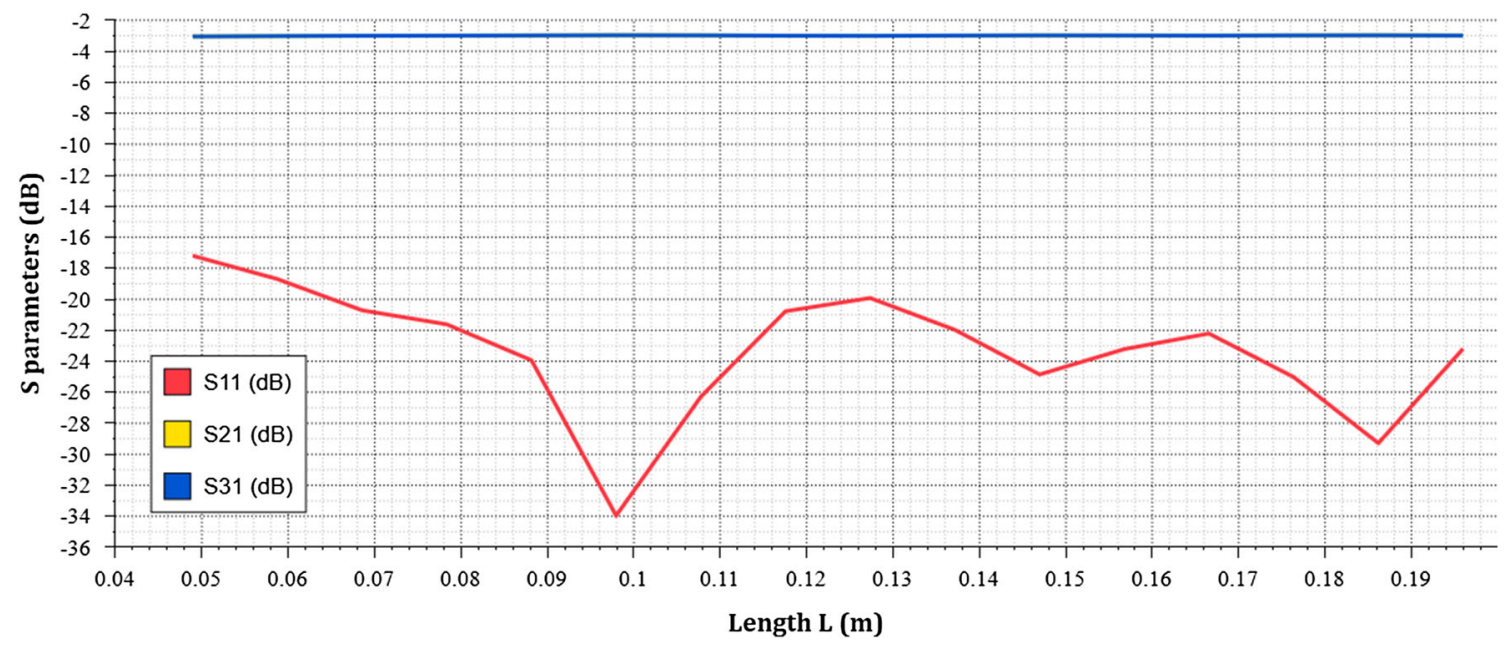

Figure 3. Dependence of $S$ parameters on variation of $L$.

$$
Z_{T E_{m 0}}=\frac{120 \pi}{\sqrt{1-\left(\frac{m \lambda}{2 a}\right)^{2}}}
$$

where $a$ is the broad dimension of the waveguide and $m$ is the number of half cycle variations in transverse plane.

Now, at the interface $3-3^{\prime}$, as discussed in section 2

$$
Z_{T E_{20}}^{\text {Transition }}=Z_{T E_{10}}^{\text {Output }} / 2 \text {. }
$$

Therefore, from Eqs. (2) and (3) we can see that

$$
\frac{120 \pi}{\sqrt{1-\left(\frac{\lambda}{a \_j n}\right)^{2}}}=\frac{120 \pi}{2 \times \sqrt{1-\left(\frac{\lambda}{2\left(a \_c o l l\right)}\right)^{2}}}
$$

Substituting the respective values in this equation, we get a_coll $=43.659 \mathrm{~mm}$ (which is $0.5381 \lambda$ at $3.7 \mathrm{GHz}$ ).

\section{Generalization and validation}

The developed methodology for obtaining the various dimensions of the power divider is readily scalable. One can scale all dimensions by $\lambda_{g}$ (at the frequency of interest) if the ratio $f / f_{c}$ is preserved, where $f_{c}$ is the cutoff frequency of the dominant mode in a waveguide. However, to obtain equal power division at any arbitrary $f / f_{c}$ ratio, a scaling by $\lambda$ is essential. Taking this into consideration, the final values of all the dimensions are as follows:

$$
\begin{aligned}
& a \_j n=1.4923 \lambda, \\
& a \_ \text {coll }=0.53841 \lambda, \\
& L=1.2333 \lambda .
\end{aligned}
$$

where $\lambda$ is in $\mathrm{m}$.

The afore-mentioned equations were tested at numerous frequencies by performing simulations in COMSOL Multiphysics, CST studio and HFSS. The resulting design data and $S_{11}, S_{21}$ and $S_{31}$ obtained in COMSOL are tabulated in table 1 . From the table, it is observed that excellent equal power division $\left(S_{21}=S_{31} \approx-3 \mathrm{~dB}\right)$ is obtained at all the test frequencies with good acceptable matching. The frequency response of the structure for the FHT designed at

\begin{tabular}{|c|c|c|c|c|c|c|c|}
\hline Waveguides & $\begin{array}{l}\text { Frequency } \\
\quad(\mathrm{GHz})\end{array}$ & $\begin{array}{c}a \_j n=1.4923 \lambda \\
(\mathrm{mm})\end{array}$ & $a_{(\mathrm{mm})}$ coll $=0.53841 \lambda$ & 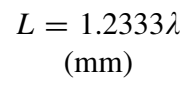 & $S_{11}(\mathrm{~dB})$ & $S_{21}(\mathrm{~dB})$ & $S_{31}(\mathrm{~dB})$ \\
\hline WR650 & 1.5 & 298.46 & 107.69 & 246.67 & -38.612 & -3.0101 & -3.0117 \\
\hline WR284 & 3.7 & 121.00 & 43.659 & 100.00 & -38.435 & -3.0112 & -3.0106 \\
\hline WR187 & 5 & 89.538 & 32.308 & 74.00 & -34.879 & -3.0125 & -3.0109 \\
\hline WR112 & 7.5 & 59.692 & 21.538 & 49.333 & -26.613 & -3.0182 & -3.0214 \\
\hline WR12 & 70 & 6.3956 & 2.3077 & 5.2857 & -27.548 & -3.019 & -3.0171 \\
\hline WR10 & 90 & 4.9743 & 1.7949 & 4.1111 & -31.329 & -3.0138 & -3.0135 \\
\hline WR6 & 150 & 2.9846 & 1.08 & 2.4667 & -38.753 & -3.0107 & -3.0115 \\
\hline WR4 & 200 & 2.2385 & 0.808 & 1.85 & -28.042 & -3.0187 & -3.0162 \\
\hline
\end{tabular}
$3.7 \mathrm{GHz}$ is depicted in figure 4. Excellent matching and power division is observed at $3.7 \mathrm{GHz}$. The structure exhibits acceptable response $\left(S_{11}<-20 \mathrm{~dB}\right)$ for a bandwidth of $100 \mathrm{MHz}$ centred around $3.7 \mathrm{GHz}$. This

Table 1. Design data and outputs for various cases. 


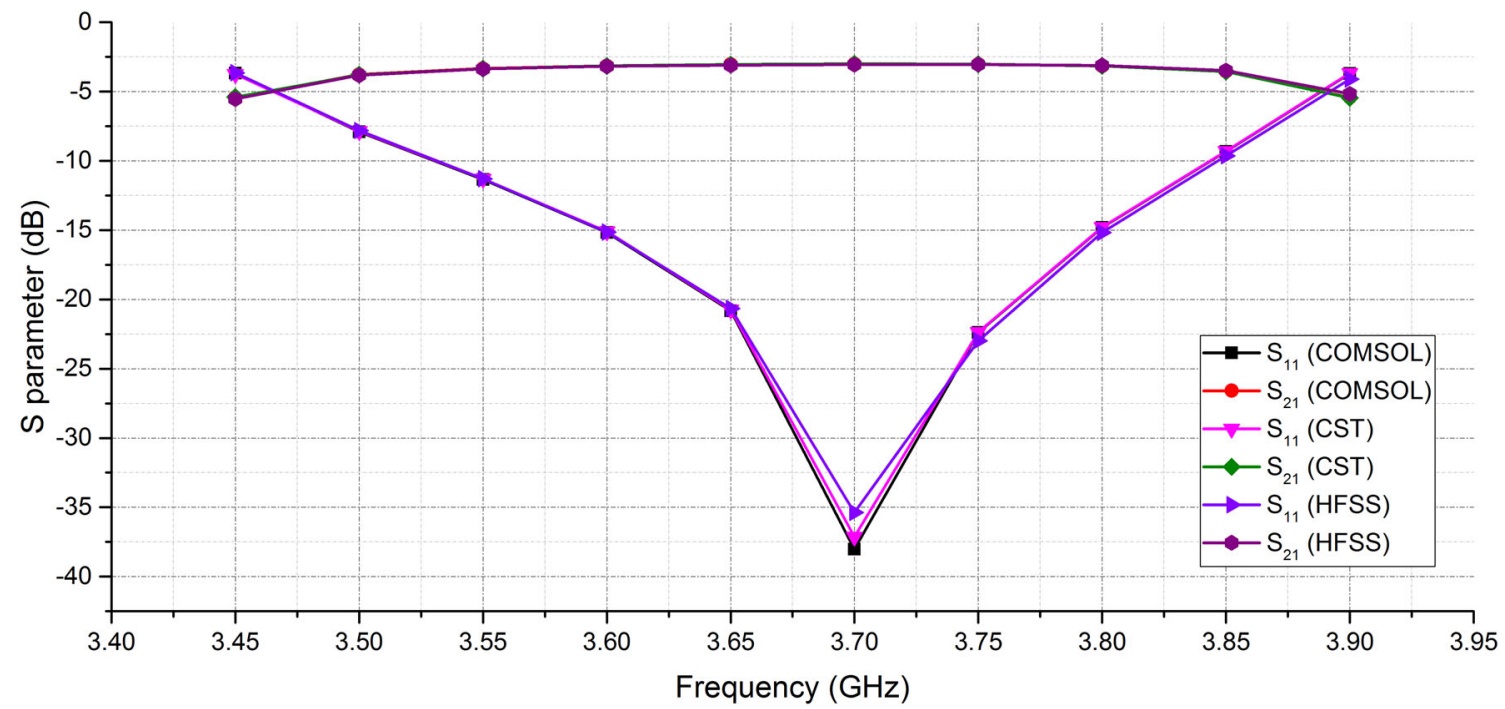

Figure 4. Frequency response of folded $\mathrm{H}$ plane tee.

bandwidth is acceptable as the klystron TH-2103D has a bandwidth of $10 \mathrm{MHz}$. The E field distribution at $500 \mathrm{~kW}$ in the FHT when excited from port 1 is presented in figure 5. The peak electric field obtained is about $7.12 \times 10^{5}$ $\mathrm{V} / \mathrm{m}$, which is well below the breakdown threshold.

\section{Thermal management}

The power lost in the waveguide due to conducting losses in its walls often leads to an increase in temperature. This attenuation due to the conducting losses for the dominant mode is given by the following Eq. [10]:

$$
\alpha_{C}=\frac{R_{S} \times\left[1+\frac{2 b}{a} \times\left(\frac{f_{C}}{f}\right)\right]}{\eta b\left[1-\left(\frac{f_{C}}{f}\right)^{2}\right]^{0.5}}
$$

where $\alpha_{C}$ is attenuation due to conduction losses, $R_{S}=\sqrt{\frac{\omega \mu}{2 \sigma}}, a$ is width of the waveguide, $b$ is height of the waveguide, $\eta$ is intrinsic impedance and $f_{C}$ is cutoff frequency of propagating mode.

From Eq. (5) and for an input power of $500 \mathrm{~kW} \mathrm{CW}$, approximately $1.3 \mathrm{~kW}$ of power would be absorbed in the walls of the proposed structure. The temperature rise of the

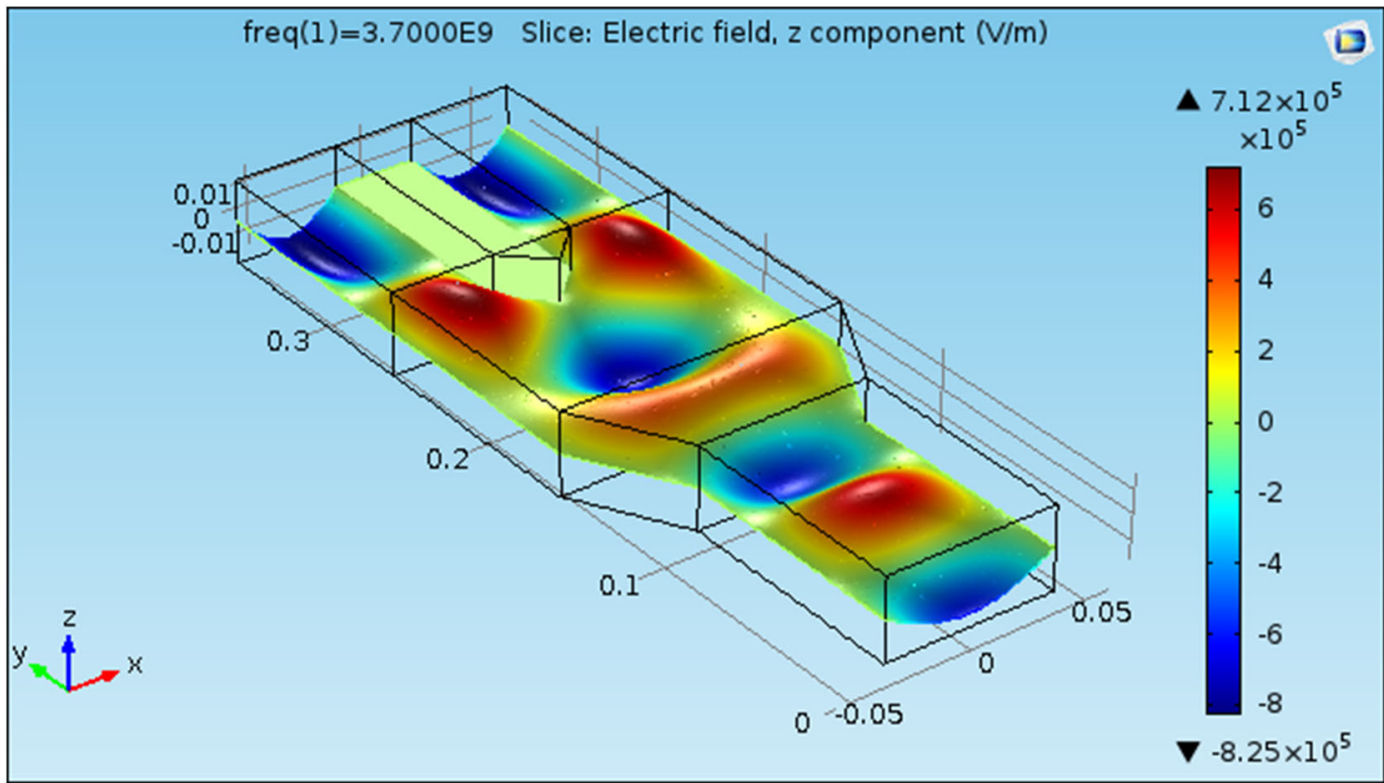

Figure 5. Electric field distribution in FHT. 
waveguide walls for input time of $1000 \mathrm{~s}$ due to this power absorption can be determined by the relation

$$
\Delta T=\frac{P \times t}{V \times C_{P} \times \rho}
$$

where $\Delta T$ is rise in temperature of the absorbing material $(\mathrm{K}), t$ is time in seconds, $V$ is volume of the absorbing material $\left(\mathrm{m}^{3}\right)$ (here, $V=6.827 \times 10^{-4} \mathrm{~m}^{3}$ for the structure with $4 \mathrm{~mm}$ thick sidewalls and $6 \mathrm{~mm}$ thick top-bottom walls), $C_{P}$ is heat capacity at constant pressure of absorbing material $(\mathrm{J} / \mathrm{kg} \mathrm{K})$ and $\rho$ is density of absorbing material $\left(\mathrm{kg} / \mathrm{m}^{3}\right)$.

Substituting all values in Eq. (6), the temperature rise comes out to be $568.50^{\circ} \mathrm{C}$. The simulation of the structure in COMSOL Multiphysics for these given parameters (at input $500 \mathrm{~kW}$ and $1000 \mathrm{~s}$ ) is shown in figure $6 \mathrm{a}$. The initial temperature of the simulated structure is $20^{\circ} \mathrm{C}$, while the final temperature as seen in figure $6 \mathrm{a}$ is $575.44^{\circ} \mathrm{C}$ (i.e., temperature rise will be $575.44^{\circ} \mathrm{C}-20^{\circ} \mathrm{C}=555.44^{\circ} \mathrm{C}$ ), which is very close to the analytical calculation done using Eq. (6). This temperature rise often cannot be tolerated in the structure and hampers the safety of its handling. Further, it also reduces the breakdown threshold. The structure was simulated to observe the stress and deformation at the high temperatures obtained. Figure $6 b$ shows the surface Von Mises stress while figure $6 \mathrm{c}$ shows the deformation of the proposed structure at $500 \mathrm{~kW}$ input for $1000 \mathrm{~s}$. As can be seen, the maximum stress obtained at certain points is
$5.49 \times 10^{9} \mathrm{~N} / \mathrm{m}^{2}$ and the maximum deformation is up to $1.28 \mathrm{~mm}$. Even small deformations, if permanent, can degrade the $S$ parameters of the component and render the component useless after repeated use. As such, forced convection cooling is employed to cool down the FHT. For this purpose, two cooling channels are placed along the $\mathrm{H}$ plane at locations where the $E$ field is maximum. This is shown in figure $7 \mathrm{a}$. Water flows through these channels at the rate of $20 \mathrm{lpm}$. The temperature profile after cooling is as depicted in figure $7 \mathrm{~b}$ for steady-state analysis. The maximum temperature reached by the structure is $51.07^{\circ} \mathrm{C}$. The outlet water temperature is $22.1^{\circ} \mathrm{C}$, while the pressure drop is 0.12 bar.

\section{Prototype fabrication and low-power testing}

A prototype FHT was fabricated in aluminium to validate the proposed methodology. The prototype was designed to operate at $3.7 \mathrm{GHz}$. All the dimensions of the $\mathrm{H}$ plane tee are as per column 2 of table 1. As both the output arms of $\mathrm{H}$ plane tee have non-standard waveguide dimensions, a transformer is attached to convert the non-standard waveguide dimension to standard WR-284 dimensions. A bend is incorporated in one of the output arms of the $\mathrm{H}$ plane tee so that VNA testing can be carried out conveniently. The other arm was fixed with a straight waveguide to equalize the output phase of both the arms. A standard
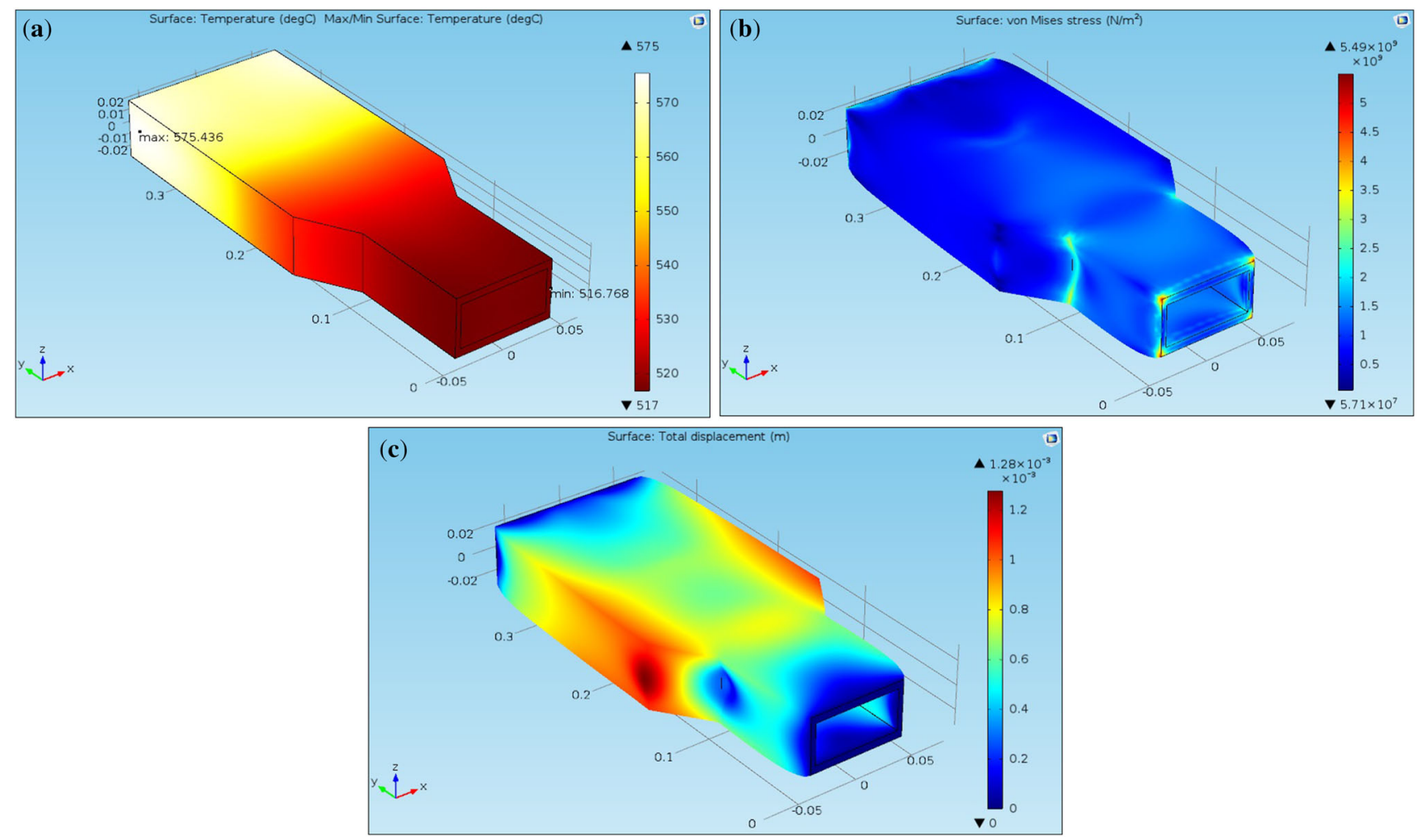

Figure 6. (a) Temperature profile without cooling, (b) surface stress of FHT and (c) surface deformation of FHT. 

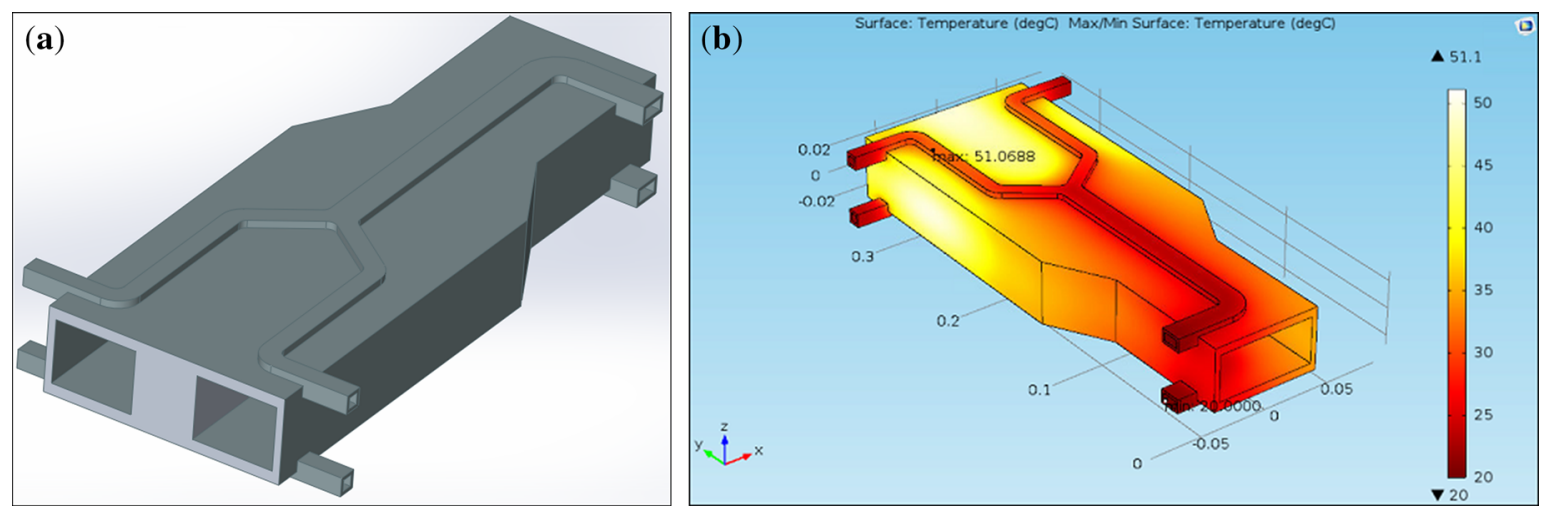

Figure 7. (a) 3D structure of the FHT with cooling lines and (b) temperature profile for $500 \mathrm{~kW}$ input to the stationary state.

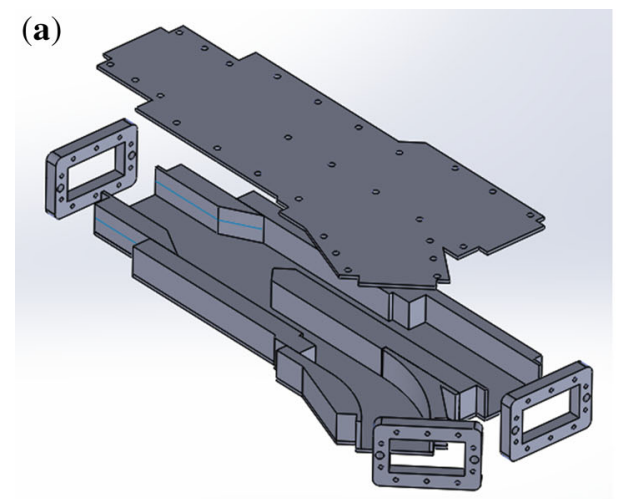

(b)

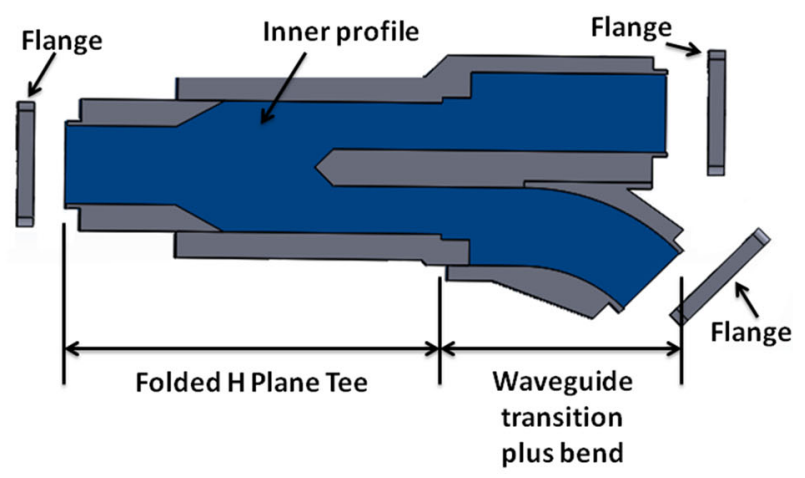

Figure 8. (a) Exploded representation of the fabricated FHT and (b) top view of the fabricated FHT without the top plate.

flange for WR-284 waveguide was used at the input and output arms to facilitate the connection of a waveguide to coaxial adapter. The signal from the adapter was then used to obtain the test results.

Water jet cutting was used to cut out an oversized top and bottom plate along with the left and right hand side profiles of the device. After the water jet cutting, all the plates were milled to their correct dimension. Finally, holes were drilled along them and they were threaded so that they could be bolted together. Figure 8a depicts the assembly of the FHT using individual sub-part while figure $8 \mathrm{~b}$ depicts the top view of the assembly.

The assembled $\mathrm{H}$ plane tee was evaluated for its RF performance by measuring its $S$ parameters using a VNA. The measurement set-up, fabricated structure and the waveguide to coaxial adapter can be seen in figure 9 . The frequency response obtained from VNA is depicted in figure 10. For the sake of convenience, the simulation results obtained from COMSOL are added in the figure for comparison. It can be seen that the measured results are in agreement with the simulated ones.

The major issue in high-power working of any waveguide component is electric breakdown due to two causes,

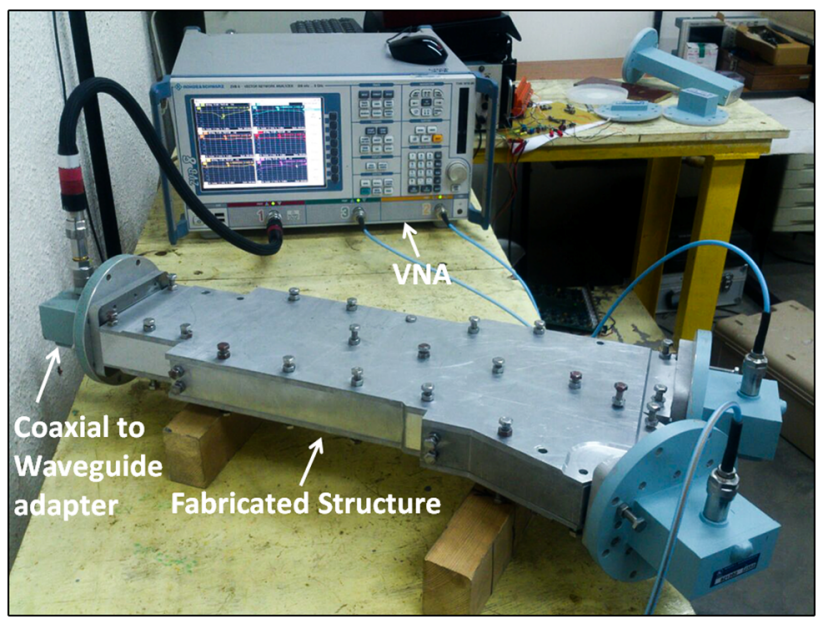

Figure 9. Measurement set-up for the FHT.

viz., high temperature and high electric field. To evaluate the effect of high temperature (due to high $\mathrm{CW}$ power) on the RF performance of the FHT, it was heated up to $60^{\circ} \mathrm{C}$ using a baking tape. This would emulate the temperature of FHT when there is a flow of approximately $500 \mathrm{~kW} \mathrm{CW}$ 


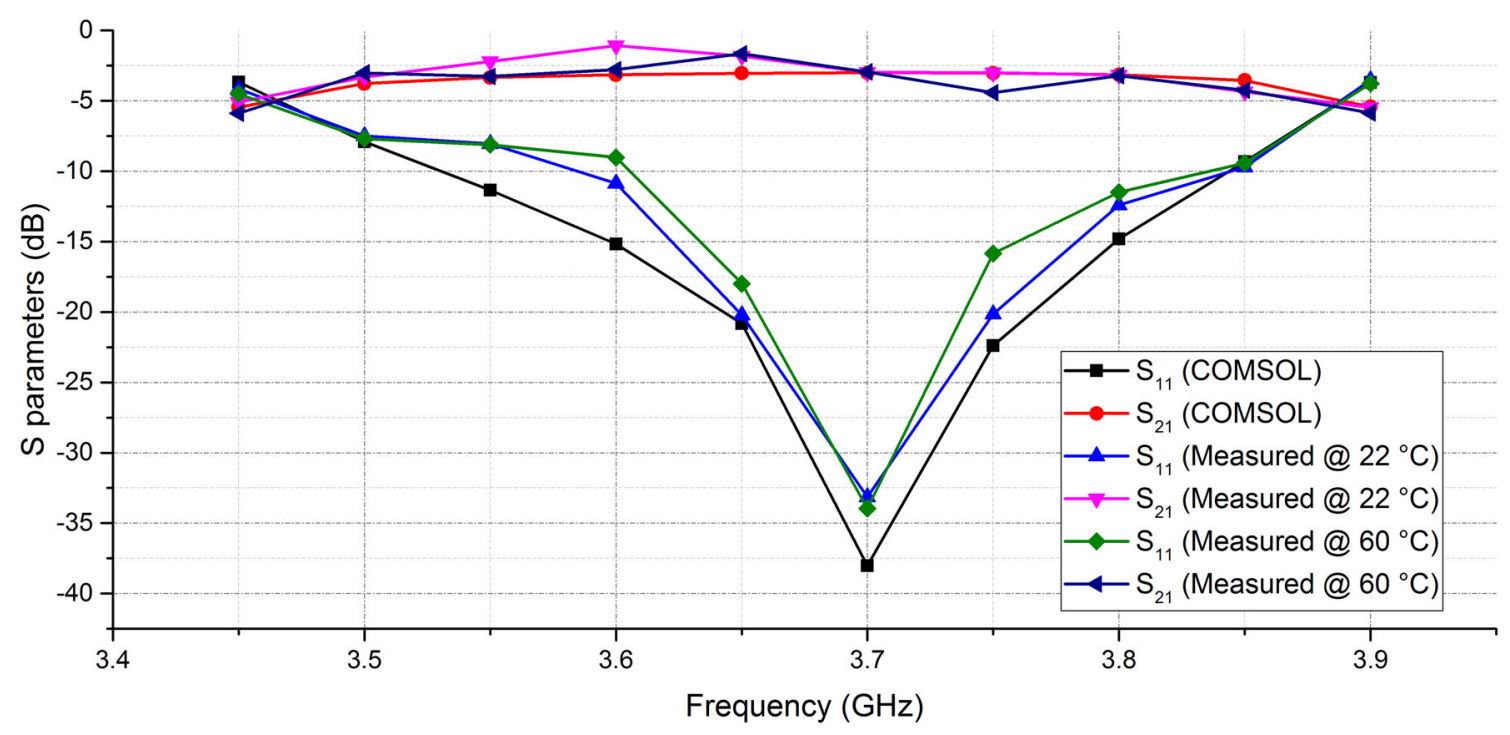

Figure 10. Comparison of simulated and measured results of the FHT.

power in the FHT, which is actively cooled with water with reference to figure $7 \mathrm{~b}$. The FHT was then tested for its RF performance on VNA. The $S$ parameters thus obtained are depicted in figure 10. It can be seen that the change in RF performance at high temperature is marginal. As the fabricated structure is only a prototype made from aluminium, high-power testing cannot be carried on it. However, if the structure is made from OFHC (Oxygen-Free High Conductivity) copper following all the protocols of constructing waveguide devices at high power like rounding off the corners, having minimum joints in the structure and pressurizing it with nitrogen to reduce the chances of multipactor breakdown, the device would work at high power as the peak electric field at $500 \mathrm{~kW} \mathrm{CW}(0.7 \mathrm{MV} / \mathrm{m})$ is an order of magnitude less than that required for breakdown for a waveguide pressurized with 3 bar of nitrogen (7 MV/ $\mathrm{m})$.

\section{Conclusion}

Table 1 shows that the proposed designing equations and methodology can give structures for a wide variety of frequency of operations. The $S$ parameters thus obtained from the FHTs at the respective frequencies are quite good and satisfactory. The widths of the output arms obtained from the proposed method are of non-standard dimensions. Thus, if standard dimensions for the designed component of all the waveguides are desired, the output arms can be transitioned to the standard dimensions by use of simple matching sections such as quarter wave transformers. The modelling of the proposed FHT structure was carried out in COMSOL Multiphysics, CST Microwave Studio and HFSS, which exhibited similar results, thus validating the correctness of the methodology. With thermal management of the structure using forced convection, cooling the designed component can be used at high $\mathrm{CW}$ power level. Finally, a prototype structure was fabricated to operate at $3.7 \mathrm{GHz}$ and the measured results were reported.

\section{Acknowledgements}

We thank the Board of Research in Nuclear SciencesPlasma and Fusion Research Committee (BRNS-PFRC) for supporting the project with Grant [39/02/2015]. We would also like to thank P R Parmar and the workshop team at Institute for Plasma Research, Gandhinagar, for all the support extended for fabricating the structure.

\section{References}

[1] Hillairet J et al 2011 RF modeling of the ITER-relevant lower hybrid antenna. Fusion Eng. Des. 86: 823-826

[2] Hillairet J et al 2012 Lower hybrid antennas for nuclear fusion experiments. In: Proceedings of the 6th European Conference on Antennas and Propagation (EUCAP), Prague, pp. 1-4

[3] Ekedahl A et al 2011 Long pulse operation with the ITERrelevant LHCD antenna in Tore Supra. AIP Conf. Proc. 1406: 399-406

[4] Ridolfini V P et al 2004 Experimental test of an ITER-like passive active multijunction lower hybrid RF launcher on the FTU Tokamak. In: Proceedings of the 31st EPS Conference on Plasma Physics, London, vol. 28G, p. 2.105

[5] Olivier J C and McNamara D A 1992 Analysis of multiport rectangular waveguide devices using pulsed finite-difference time-domain (FDTD) technique. Electron. Lett. 28(2): 129-131 
[6] Panda D K and Chakraborty A 2008 Analysis of folded H-plane tee junction using multiple cavity modeling technique. In: Proceedings of the IEEE Region 10 and the Third International Conference on Industrial and Information Systems, vol. 214, pp. 1-6

[7] Yang S and Fathy A E 2009 Design equations of arbitrary power split ratio waveguide $\mathrm{T}$-junctions using a curve fitting approach. Int. J. RF Microw. Comput. Aided Eng. 19(1): 91-98

[8] Marcuvitz N 1951 Waveguide handbook. New York: McGraw-Hill Book Company

[9] Ragan G L 1948 Microwave transmission circuits. New York: McGraw-Hill Book Company

[10] Pozar D M 2009 Microwave engineering. Hoboken: Wiley 